\title{
The COVID-19 pandemic and diabetes mellitus
}

\author{
Athanasia K. Papazafiropoulou, Stavros Antonopoulos
}

$1^{\text {st }}$ Department of Internal Medicine and Diabetes Center, Tzaneio General Hospital of Piraeus, Piraeus, Greece

Submitted: 23 May 2020

Accepted: 28 May 2020

Arch Med Sci Atheroscler Dis 2020; 5: e200-e205

DOI: https://doi.org/10.5114/amsad.2020.97435

Copyright (c) 2020 Termedia \& Banach

\section{Abstract}

COVID-19, a disease caused by a novel coronavirus, SARS-CoV-2, has reached the proportion of a pandemic and presents with either mild and moderate symptoms or in severe cases with acute respiratory distress syndrome, multiple organ dysfunction syndrome and even death. Older age, hypertension, cardiovascular disease, diabetes mellitus and obesity significantly increase morbidity and mortality in COVID-19 patients. In the present review we summarize the existing, and daily growing, data on the impact of COVID-19 infection on patients with diabetes, their antidiabetic therapy as well as the extra precautions, apart from good glucose control, they have to take in order not to contract the virus. Social distancing and strict hand hygiene are of great importance in order to help the global goal of eradication of the disease.

Key words: COVID-19, SARS-CoV-2, diabetes, social distancing, angiotensin I-converting enzyme, dipeptidyl peptidase-4.

\section{Introduction}

In December 2019, a pneumonia of unknown origin with high fatality, mostly due to acute respiratory distress syndrome (ARDS), was observed in Wuhan, China. On February 11, 2020 the cause of the disease was discovered and named SARS-CoV-2 by the International Committee on Taxonomy of Viruses [1, 2]. SARS-CoV-2 belongs to the coronaviruses, like severe acute respiratory syndrome coronavirus (SARS-CoV) and middle-east respiratory syndrome coronavirus (MERS-CoV), which are named for the crown-like spikes on their surface. SARS-CoV-2 is more infectious, with over 80,000 cases in China and nearly 2,800,000 cases worldwide reported as of April 25, 2020, according to the Center for Systems Science and Engineering at John Hopkins University [3]. Most COVID-19 patients present with mild and moderate symptoms, but severe cases can present with ARDS, multiple organ dysfunction syndrome (MODS) and even death. It has been reported that the fatality rate of COVID-19 varies from $1.4 \%$ to $4.3 \%$ in different regions or hospitals [4, 5].

Diabetes, and its complications, is one of the leading causes of morbidity and mortality worldwide [6]. Patients with diabetes are at high risk of infections, especially influenza and pneumonia [7, 8]. Chronic hyperglycemia that characterizes patients with diabetes leads to impaired phagocytosis by neutrophils, macrophages and monocytes, impaired neutrophil chemotaxis and bactericidal activity, and impaired innate cell-mediated immunity $[7,8]$. Therefore, in order to protect patients

\author{
Corresponding author: \\ Athanasia K. \\ Papazafiropoulou \\ MD, MSc, PhD \\ $1^{\text {st }}$ Department \\ of Internal Medicine \\ and Diabetes Center, \\ Tzaneio General \\ Hospital of Piraeus \\ 1 Zanni and Afentouli St \\ Piraeus 18536, Greece \\ Phone: +30 697996483 \\ E-mail: \\ pathan@ath.forthnet.gr
}


with diabetes from influenza and pneumonia, pneumococcal and annual influenza vaccinations are recommended to all patients with diabetes aged above 2 years. In addition, uncontrolled glycemia is a significant risk factor of severity and death in patients with diabetes who are infected by different viruses [9-11]. During the last three pandemics, Influenza A 2009 (H1N1), SARS coronavirus and MERS-CoV, diabetes per se was an important risk factor for morbidity and mortality [9-11]. This is also confirmed in the present pandemic of COVID-19, as will be discussed in the next sections.

Therefore, the aim of the present review is to summarize the existing literature data on the epidemiology of COVID-19 in patients with diabetes, the possible pathogenetic mechanisms linking the two diseases, and the potential effects of antidiabetic agents on the infection.

\section{Epidemiology of COVID-19 in patients with diabetes}

It is very interesting that data on the prevalence of diabetes in patients with COVID-19 diabetes are growing day by day and are different depending on the studied population.

Three recent meta-analyses showed that the most prevalent comorbidities among patients with COVID-19 were hypertension and diabetes, followed by cardiovascular diseases and respiratory system disease [12-14]. The first one, including 8 studies with 46,248 infected patients, demonstrated a prevalence of diabetes of about $8 \pm 6 \%$ (95\% confidence interval $(\mathrm{Cl}): 6-11 \%$ ) [12]. The second one was a comprehensive systematic search on articles published until 15 February 2020 and included data of 76,993 patients from 10 different studies [13]. According to this metaanalysis, the prevalence of diabetes in people infected with SARS-CoV-2 was estimated as $7.87 \%$ (95\% Cl: 6.57-9.28\%). Another meta-analysis conducted to estimate the prevalence and impact of cardiovascular metabolic diseases on COVID-19 in China included six studies with 1,527 patients [14]. The proportions of hypertension, cardio/ cerebrovascular disease and diabetes in patients with COVID-19 were $17.1 \%, 16.4 \%$ and $9.7 \%$, respectively. The incidences of hypertension, cardio/ cerebrovascular diseases and diabetes were about twofold, threefold and twofold, respectively, higher in intensive care unit (ICU)/severe cases than in their non-ICU/severe counterparts.

Even higher prevalence of diabetes in people infected with SARS-CoV-2 was found in studies from Wuhan, the center of the disease. A study by Zhou et al. [15], in 191 patients with COVID-19, showed that almost half of them had a comorbidity, with hypertension being the most common (30\% pa- tients), followed by diabetes (19\% patients) and coronary heart disease ( $8 \%$ patients). In another study in 85 patients, $68.2 \%$ had one or more comorbidities, with hypertension (37.6\%), diabetes (22.4\%) and coronary heart disease (11.8\%) being the most common comorbidities [16]. In a study by Wan et al. [17], of 135 hospitalized patients with COVID-19 who were enrolled, $31.9 \%$ of patients had underlying disease, primarily hypertension (9.6\%), diabetes $(8.9 \%)$, cardiovascular disease (5.2\%), and malignancy (3.0\%). Guan et al. analyzed the data from 1,590 laboratory-confirmed hospitalized patients with COVID-19 from 575 hospitals in China between December $11^{\text {th }}, 2019$ and January $31^{\text {st }}, 2020$. According to their findings, the most prevalent comorbidity was hypertension (16.9\%), followed by diabetes (8.2\%). Diabetes (hazard ratio (HR): $1.59,95 \% \mathrm{Cl}: 1.03-2.45)$ was a risk factor of mortality [18].

A study by Yang et al. [19], in 32 non-survivors from a group of 52 ICU patients with COVID-19, found that the most common comorbidities were cerebrovascular diseases (22\%) and diabetes (22\%). Another study [20], which included 1,099 patients with COVID-19, showed that in 173 with severe disease the most common comorbidities were hypertension (23.7\%), diabetes (16.2\%), coronary heart (5.8\%) and cerebrovascular disease (2.3\%). In a third study [21], in 140 patients who were admitted to hospital with COVID-19, 30\% had hypertension and $12 \%$ diabetes, while diabetes was not a risk factor for severe disease course.

Presence of diabetes in patients with COVID-19 was associated with the worst outcomes. Diabetes was present in $42.3 \%$ of 26 fatalities due to COVID-19 in Wuhan, China [22]. Wu et al. showed that in 201 patients with COVID-19 prevalence of diabetes among patients who developed ARDS, compared with those who did not, was 19.0\% compared to $5.1 \%$, respectively [23]. Another study that estimated clinical features of deaths in the novel COVID-19 epidemic in China found a significant difference in the proportion of diabetes between the deceased patients (26.2\%) and the Hubei population (5.6\%) [24]. The authors suggested that diabetes might be associated with increased risk of mortality.

Since March $19^{\text {th }} 2020$, when Italy was the country second most affected by COVID-19, new data on the prevalence of diabetes among patients in Europe have been added to the literature. At the University Hospital of Padova, among 146 hospitalized patients with confirmed COVID-19, 13 had pre-existing diabetes, yielding a prevalence of $8.9 \%$ (95\% Cl: 5.3-14.6) [25]. Even higher diabetes prevalence was recorded by another two studies. A study by the Istituto Superiore di Sanita reported that among 355 deceased patients with available information on comorbidities, diabetes 
prevalence was $35.5 \%$ [26]. In 2018, diabetes prevalence among Italian citizens with the same age range and sex distribution was $20.3 \%$ [27]. Thus, the rate ratio of diabetes among patients who died with SARS-CoV-2 infection compared to the general population was 1.75 .

An analysis on 122,653 U.S. COVID-19 cases reported to the Centers for Disease Control and Prevention as of March 28, 2020, showed that underlying health conditions, including, diabetes mellitus, hypertension, chronic obstructive pulmonary disease, coronary artery disease, cerebrovascular disease, chronic renal disease, and smoking, are risk factors for severe disease or death from COVID-19 [20, 28]. Finally, a study in the U.S.A. in patients from 9 Seattle-area hospitals who were admitted to the ICU with confirmed infection with severe COVID-19 showed that $58 \%$ of patients had diabetes mellitus [29]. In 5,700 patients with COVID-19 admitted to 12 hospitals in New York the most common comorbidities were hypertension (56.6\%), obesity (41.7\%), and diabetes (33.8\%) [30]. Another publication analyzing data of COVID-19-associated hospitalization rates for patients admitted during March 2020, the first month of U.S. surveillance, showed that the most common underlying conditions were hypertension (49.7\%), obesity (48.3\%), chronic lung disease (34.6\%), diabetes mellitus (28.3\%), and cardiovascular disease (27.8\%) [31].

In conclusion, diabetes is a risk factor for COVID-19 and, in addition, is associated with increased mortality, as has been confirmed by a recent metanalysis [32]. Among 1,382 patients, diabetes was the second more frequent comorbidity while diabetic patients had a significantly increased risk of ICU admission (OR $=2.79$, $95 \% \mathrm{Cl}: 1.85-4.22)$ and a higher mortality risk $(\mathrm{OR}=3.21,95 \% \mathrm{Cl}: 1.82-5.64)$ [32]. Finally, in the current COVID-19 pandemic, reports from China, Italy and the U.S.A. showed that age is a significant risk factor for morbidity and mortality, in addition to diabetes per se, with older patients with diabetes being at higher risk for severe disease and mortality [20, 33, 34].

\section{Pathogenetic mechanisms linking COVID-19 with diabetes: role of angiotensin-converting enzyme}

It is known that diabetes, especially type $2 \mathrm{di}$ abetes, is characterized by the presence of lowgrade chronic inflammation induced by the excessive visceral adipose tissue. Chronic inflammation combined with hyperglycemia results in an abnormal and ineffective immune response, including decreased mobilization of polymorphonuclear leukocytes, chemotaxis, and phagocytic activity, lower secretion of cytokines such as interleukin-1
(IL-1) and IL-6, and glycation of immunoglobulin $[35,36]$. IL-6, along with fibrinogen, C-reactive protein, and D-dimer, are found to be more elevated in COVID-19 cases with than in those without diabetes [35, 36].

Apart from impaired neutrophil chemotaxis and phagocytosis, there are several specific factors responsible for increased risk and severity of COVID-19 in diabetes. SARS-CoV-2, like SARS-CoV, utilizes angiotensin-converting enzyme (ACE) 2 as a receptor for entry into the cell [37]. This enzyme is a homologue of ACE1, which converts angiotensin I to angiotensin II, and is therapeutically targeted in hypertension and heart failure. ACE2 is widely expressed, including in the lungs, cardiovascular system, gut, kidneys, central nervous system, and adipose tissue. The S-glycoprotein on the surface of SARS-CoV2 binds to ACE2 and causes a conformational change in the S-glycoprotein that induces proteolytic digestion by host cell proteases, leading to internalization of the virion [38]. Cellular entry of the virus triggers an inflammatory response leading to a 'cytokine storm' which could lead to organ damage and multi-organ failure seen in severe disease [38]. Diabetic mice have been found to have increased expression of ACE2 in the renal cortex, liver and pancreas, but not in the lungs [39]. Amongst others, pioglitazone and liraglutide have also been shown to be associated with ACE2 upregulation in animal studies [40, 41].

Furthermore, diabetes more often co-exists with hypertension, and treatment with either ACE inhibitors, or angiotensin-receptor blockers (ARBs) is very common. Therefore, one could assume that ACE inhibitors and/or ARBs might have deleterious effects in patients with diabetes and COVID-19. ACE inhibitors inhibit ACE, leading to decreased angiotensin I levels, causing a possible negative feedback loop that ultimately upregulates more ACE2 receptor to be able to interact with the decreased angiotensin I substrate available [42]. In addition, plasma levels of angiotensin II increase with ARB dosing [43]. Angiotensin II is a known substrate for ACE2 and might result in increasing the expression of the linked enzyme. This ACE2 receptor upregulation results in increased binding sites for SARS-CoV-2, leading to preferential COVID-19. On the other hand, treatment with ARBs has been proposed as a potential therapeutic strategy for COVID-19 [44], supported by observations that blocking the renin-angiotensin system in animal models attenuated the lung injury caused by SARS-CoV, which also utilizes ACE2 [45].

However, a study by Peng et al., among 112 patients, showed that most deaths occurred secondarily to fulminant inflammation, lactic acidosis, and thrombotic states [46] while ACE inhibitor and ARB therapy was not associated with morbid- 
ity or mortality [46]. Another study, in 1,178 hospitalized patients with COVID-19 infections at the Central Hospital of Wuhan, suggested that ACE inhibitors/ARBs are not associated with the severity or mortality of COVID-19 in such patients [47]. The percentage of patients with hypertension taking ACE inhibitors/ARBs did not differ between those with severe and non-severe infections (32.9\% vs. $30.7 \%, p=0.645)$; nor did it differ between non-survivors and survivors $(27.3 \%$ vs. $33.0 \%$, $p=0.34$ ) [47]. A recent position statement from the European Society of Cardiology recommends that patients continue treatment with their usual anti-hypertensive treatment and that there is no clinical or scientific evidence to suggest that treatment with ACE inhibitors or ARBs should be discontinued because of COVID-19 [48].

\section{Role of antidiabetic agents in COVID-19}

There are no data on the differential effects of oral antidiabetic drugs on the disease course in COVID-19. In general, previous antidiabetic agents in patients with mild COVID-19 should be evaluated and followed as appropriate. For severe and critically ill patients, intravenous insulin therapy, including rapid-acting prandial/basal insulin, should be the preferred therapy option.

During the COVID-19 pandemic, the relationship of coronavirus to the cellular type II transmembrane protein dipeptidyl peptidase-4 (DPP-4) (CD26) has generated great interest since a lot of patients with diabetes are under DPP4 inhibitors therapy. It is known that DPP4 inhibitors target the enzymatic activity of DPP4, a type II transmembrane glycoprotein, expressed ubiquitously in many tissues, including immune cells [49]. Apart from breaking down circulating glucagon-like peptide-1 (GLP-1), DPP4 activates T-cells, and upregulates CD86 expression and the NF- $\kappa B$ pathway, thereby promoting inflammation. DPP4 serves as the receptor for MERS-CoV, in the same way as ACE2 is the receptor for SARS-CoV and SARS-CoV2 [50, 51], and, therefore, DPP-4 inhibitors might reduce the viral entry of MERS-CoV. However, an in vitro study, using sitagliptin, vildagliptin and saxagliptin, failed to stop coronavirus viral entry into the cells [52].

Regarding the newer antidiabetic agents, GLP-1 receptor analogues have shown significant anti-inflammatory and anti-adipogenic effects [53, 54], but no data exist regarding their effect on patients with COVID-19. Similar evidence on the effect on inflammation is also available for the sodium-glucose cotransporter-2 (SGLT-2) inhibitors [55]. However, SGLT-2 inhibitors should be used with caution in patients with COVID-19 since there is the fear of euglycemic diabetic ketoacidosis in case of dehydration. Finally, a new random- ized, global trial, named DARE-19, will evaluate dapagliflozin's ability to reduce the risk of disease progression, clinical complications, and death from COVID-19 in patients with cardiovascular, metabolic or kidney risk factors [56].

Finally, the anti-malarial hydroxychloroquine has been used against COVID-19. The drug acts by raising intracellular $\mathrm{pH}$, which inhibits enzymatic degradation of insulin, resulting in recirculation of a substantial proportion of insulin in the active form $[57,58]$. It has been known for more than 30 years that hydroxychloroquine has hypoglycemic effects that might cause hypoglycemia. Therefore, in the case of co-administration of hydroxychloroquine together with other anti-diabetic drugs, the dosages of concomitant therapies may be reassessed, particularly in patients at higher hypoglycemic risk $[57,58]$.

\section{Management of diabetes in the COVID-19 era}

During the COVID-19 pandemic, diabetes management can be challenging. Some general recommendations regarding the management of diabetes per se and for patients with diabetes and COVID-19 have been published by the American Diabetes Association [59]. In general, patients with diabetes have to drink lots of water and fluids in order to avoid dehydration, have to maintain glycemic control, and have to monitor blood sugar levels more frequently in order to avoid hypoglycemic episodes and ketoacidosis. Furthermore, patients with diabetes have to establish and maintain strict personal hygiene, such as washing hands and cleaning the injection/infusion and finger-stick sites with soap and water or rubbing alcohol.

Finally, the optimization of physician-patient communication for diabetes management in the era of social distancing, isolation, and quarantine must be emphasized. As long as patients have access to technology, their communication with their health care providers can be maintained quite unobstructed, even if it is related to mild to moderate symptoms of COVID-19 infection that can be managed at home or it is related to their diabetes management [60]. More important, this can be done without the need for physical office visits.

\section{Conclusions}

Most of the available studies have shown that diabetes as a distinctive comorbidity is associated with more severe disease, acute respiratory distress syndrome and increased mortality. Hence, following current recommendations for the general population, social distancing, and strict hand and respiratory hygiene are of great importance in order to help the global goal of eradication of the disease. Finally, and always looking at the bright 
side of things, the new era that already has started is giving us the opportunity of new ways of communication between patients with diabetes and health care providers that can be established and used also after the COVID-19 pandemic.

\section{Conflict of interest}

The authors declare no conflict of interest.

\section{References}

1. Zhu N, Zhang D, Wang W, et al.; China Novel Coronavirus I, Research T. A novel coronavirus from patients with pneumonia in China. N Engl J Med 2020; 382: 727-33.

2. Chan JF, Yuan S, Kok KH, et al. A familial cluster of pneumonia associated with the 2019 novel coronavirus indicating person-to-person transmission: a study of a family cluster. Lancet 2020; 395: 514-23.

3. John Hopkins University of Medicine: Coronavirus Resource Center. https://coronaviru s.jhu.edu/map.html. Accessed 25 April 2020.

4. Chen N, Zhou M, Dong X, et al. Epidemiological and clinical characteristics of 99 cases of 2019 novel coronavirus pneumonia in Wuhan, China: a descriptive study. Lancet 2020; 395: 507-13.

5. Wang D, Hu B, Hu C, et al. Clinical characteristics of 138 hospitalized patients with 2019 novel coronavirus-infected pneumonia in Wuhan, China. JAMA 2020; 232: 1061-9.

6. Williams R, Karuranga S, Malanda B, et al. Global and regional estimates and projections of diabetes-related health expenditure: Results from the International $\mathrm{Di}$ abetes Federation Diabetes Atlas, 9th edition. Diabetes Res Clin Pract 2020. DOI:https://doi.org/10.1016/j.diabres.2020.108072.

7. McDonald HI, Nitsch D, Millett ER, Sinclair A, Thomas SL. New estimates of the burden of acute communityacquired infections among older people with diabetes mellitus: a retrospective cohort study using linked electronic health records. Diabet Med 2014; 31: 606-14.

8. Li S, Wang J, Zhang B, Li X, Liu Y. Diabetes mellitus and cause-specific mortality: a population-based study. Diabetes Metab J 2019; 43: 319-41.

9. Yang JK, Feng Y, Yuan MY, et al. Plasma glucose levels and diabetes are independent predictors for mortality and morbidity in patients with SARS. Diabet Med 2006; 23: 623-8.

10. Schoen K, Horvat N, Guerreiro NFC, de Castro I, de Giassi KS. Spectrum of clinical and radiographic findings in patients with diagnosis of $\mathrm{H} 1 \mathrm{~N} 1$ and correlation with clinical severity. BMC Infect Dis 2019; 19: 964.

11. Song Z, Xu Y, Bao L, et al. From SARS to MERS, Thrusting Coronaviruses into the Spotlight. Viruses 2019; 11. pii: E59.

12. Yang J, Zheng Y, Gou X, et al. Prevalence of comorbidities and its effects in coronavirus disease 2019 patients: a systematic review and meta-analysis. Int J Infect Dis 2020; 94: 91-5.

13. Emami A, Javanmardi F, Pirbonyeh N, Akbari A. Prevalence of underlying diseases in hospitalized patients with COVID-19: a systematic review and meta-analysis. Arch Acad Emerg Med 2020; 8: e35.

14. Li B, Yang J, Zhao F, et al. Prevalence and impact of cardiovascular metabolic diseases on COVID-19 in China. Clin Res Cardiol 2020; 109: 531-8.
15. Zhou F, Yu T, Du R, et al. Clinical course and risk factors for mortality of adult inpatients with COVID-19 in Wuhan, China: a retrospective cohort study. Lancet 2020; 395: 1054-62.

16. Du Y, Tu L, Zhu P, et al. Clinical features of 85 fatal cases of COVID-19 from Wuhan: a retrospective observational study. Am J Respir Crit Care Med 2020; 201: 1372-9.

17. Wan S, Xiang Y, Fang W, et al. Clinical features and treatment of COVID-19 patients in northeast Chongqing. J Med Virol 2020; 201: 1372-9.

18. Guan WJ, Liang WH, Zhao Y, et al.; China Medical Treatment Expert Group for Covid-19. Comorbidity and its impact on 1590 patients with Covid-19 in China: a nationwide analysis. Eur Respir J 2020; 55: 2000547.

19. Yang $X, Y u$ Y, Xu J, et al. Clinical course and outcomes of critically ill patients with SARS-CoV-2 pneumonia in Wuhan, China: a single-centered, retrospective, observational study. Lancet Respir Med 2020; 8: 475-81.

20. Guan W, Ni Z, Hu Y, et al.; China Medical Treatment Expert Group for Covid-19. Clinical characteristics of coronavirus disease 2019 in China. N Engl J Med 2020; 382: 1708-20.

21. Zhang JJ, Dong X, Cao YY, et al. Clinical characteristics of 140 patients infected by SARS-CoV-2 in Wuhan, China. Allergy 2020; 75: 1730-41.

22. Deng SQ, Peng HJ. Characteristics of and public health responses to the Coronavirus disease 2019 outbreak in China. J Clin Med 2020; 9: 575.

23. Wu C, Chen X, Cai Y, et al. Risk factors associated with acute respiratory distress syndrome and death in patients with coronavirus disease 2019 pneumonia in Wuhan, China. JAMA Intern Med 2020. doi:10.1001/ jamainternmed.2020.0994.

24. Leung C. Clinical features of deaths in the novel coronavirus epidemic in China. Rev Med Virol 2020; 30: e2103.

25. Longato E, Di Camillo B, Sparacino G, Saccavini C, Avogaro A, Fadini GP. Diabetes diagnosis from administrative claims and estimation of the true prevalence of diabetes among 4.2 million individuals of the Veneto region (North East Italy). Nutr Metab Cardiovasc Dis 2020; 30: 84-91.

26. Istituto Superiore di Sanita: Report of characteristics of patients died positive for COVID-19 in Italy. https:// www.epice ntro.iss. it/coron aviru s/bolle ttino /Repor t-COVID -2019_17_marzo -v2. pdf Article in Italian, last accessed on March $19^{\text {th }}, 2020$.

27. ARNO Diabetes Observatory: Healthcare profile oftheltalian diabetic population. https://www.sidita lia.it/clinica/ linee-guida-societari/send/80-linee-guida-documentisocietari/5025-rapporto-arno-diabete-2019. Accessed 19 Mar 2019.

28. COVID-19 Surveillance Group. Characteristics of COVID-19 patients dying in Italy: report based on available data on March $20^{\text {th }}, 2020$. Rome, Italy: Instituto Superiore Di Sanita; 2020. https://sci-hub.tw/https:// www.epicentro.iss.it/coronavirus/bollettino/ReportCOVID-2019_20_marzo_eng.pdfpdf icon.

29. Bhatraju PK, Ghassemieh BJ, Nichols M, et al. Covid-19 in critically ill patients in the seattle region - case series. N Engl J Med 2020; 382: 2012-22.

30. Richardson S, Hirsch JS, Narasimhan M, et al. Presenting characteristics, comorbidities, and outcomes among 5700 patients hospitalized with COVID-19 in the New York City Area. JAMA 2020; 323: 2052-9.

31. Garg S, Kim L, Whitaker M, et al. hospitalization rates and characteristics of patients hospitalized with laboratory-confirmed coronavirus disease 2019 - COVID-NET, 
14 States, March 1-30, 2020. Morb Mortal Wkly Rep 2020; 69: 458-64.

32. Wu Z, McGoogan JM. Characteristics of and important lessons from the coronavirus disease 2019 (COVID-19) outbreak in China: summary of a report of 72314 cases from the chinese center for disease control and prevention. JAMA 2020; 323: 1239-42.

33. Onder G, Rezza G, Brusaferro S. Case-fatality rate and characteristics of patients dying in relation to COVID-19 in Italy. JAMA 2020. doi: 10.1001/jama.2020.4683.

34. Roncon L, Zuin M, Rigatelli G, Zuliani G. Diabetic patients with COVID-19 infection are at higher risk of ICU admission and poor short-term outcome. J Clin Virol 2020; 127: 104354.

35. Mehta P, McAuley DF, Brown M, Sanchez E, Tattersall RS, Manson JJ; HLH Across Speciality Collaboration, UK. COVID-19: consider cytokine storm syndromes and immunosuppression. Lancet 2020; 395: 1033-4.

36. Perez-Moreiras JV, Gomez-Reino JJ, Maneiro JR, et al.; Tocilizumab in Graves Orbitopathy Study Group. Efficacy of tocilizumab in patients with moderate-to-severe corticosteroid-resistant graves orbitopathy: a randomized clinical trial. Am J Ophthalmol 2018; 195: 81-190.

37. Vaduganathan M, Vardeny O, Michel T, McMurray JJV, Pfeffer MA, Solomon, SD. Renin-angiotensin-aldosterone system inhibitors in patients with Covid-19. N Eng J Med 2020; 382: 1653-9.

38. Hoffmann $M$, Kleine-Weber $\mathrm{H}$, Schroeder $\mathrm{S}$, et al. SARSCoV-2 cell entry depends on ACE2 and TMPRSS2 and is blocked by a clinically proven protease inhibitor. Cell 2020; 181: 271-80.

39. Roca-Ho H, Riera M, Palau V, Pascual J, Soler MJ. Characterization of ACE and ACE2 287 expression within different organs of the NOD mouse. Int J Mol Sci 2017; 18: E563.

40. Fang L, Karakiulakis G, Roth M. Are patients with hypertension and diabetes mellitus at increased risk for COVID-19 infection? Lancet Respir Med 2020; 8: e21.

41. Romaní-Pérez M, Outeiriño-Iglesias V, Moya CM, et al. Activation of the GLP-1 receptor by liraglutide increases ACE2 expression, reversing right ventricle hypertrophy, and improving the production of SP-A and SP-B in the lungs of type 1 diabetes rats. Endocrinology 2015; 156: 3559-69.

42. Nahum LH. The renin angiotensin-aldosterone system (RAAS) in normal man. Conn Med 1965; 29: 710-1.

43. Gavras I, Gavras H. Effects of eprosartan versus enalapril in hypertensive patients on the renin-angiotensin-aldosterone system and safety parameters: results from a 26-week, double-blind, multicentre study. Eprosartan Multinational Study Group. Curr Med Res Opin 1999; 15: 15-24.

44. Zhang H, Penninger JM, Li Y, Zhong N, Slutsky AS. Angiotensin converting enzyme 2 (ACE2) as a SARS-CoV-2 receptor: molecular mechanisms and potential therapeutic target. Intensive Care Med 2020; 46: 586-90.

45. Imai Y, Kuba K, Rao S, et al. Angiotensin converting enzyme 2 protects from severe acute lung failure. Nature 2005; 436: 112-6.

46. Peng YD, Meng K, Guan HQ, et al. Clinical characteristics and outcomes of 112 cardiovascular disease patients infected by 2019-nCoV. Zhonghua Xin Xue Guan Bing Za Zhi 2020; 48: E004.

47. Li J, Wang X, Chen J, Zhang H, Deng A. Association of renin-angiotensin system inhibitors with severity or risk of death in patients with hypertension hospitalized for coronavirus disease 2019 (COVID-19) infection in Wu- han, China. JAMA Cardiol 2020 doi: 10.1001/jamacardio.2020.1624.

48. de Simone G, ESC Council on Hypertension. Position Statement of the ESC Council on Hypertension on ACE-Inhibitors and Angiotensin Receptor Blockers. European Society of Cardiology. 13 March 2020. Available at https://www.escard io.org/Councils/Council-onHypertension-(CHT)/News/positionstatement-of-theesc-council-on-hypertension-on-ace-inhibitorsand-ang.

49. lacobellis G. COVID-19 and diabetes: can DPP4 inhibition play a role? Diabetes Res Clin Pract 2020; 162: 108125.

50. Gierer S, Bertram S, Kaup F, et al. The spike protein of the emerging betacoronavirus EMC uses a novel coronavirus receptor for entry, can be activated by TMPRSS2, and is targeted by neutralizing antibodies. J Virol 2013; 87: 5502-11.

51. Qian Z, Dominguez SR, Holmes KV. Role of the spike glycoprotein of human Middle East respiratory syndrome coronavirus (MERS-CoV) in virus entry and syncytia formation. PLoS One 2013; 8: e76469.

52. Raj VS, Mou H, Smits SL, et al. Dipeptidyl peptidase 4 is a functional receptor for the emerging human coronavirus-EMC. Nature 2013; 495: 251-4.

53. Rizzo M, Nikolic D, Banach M, Patti AM, Montalto G, Rizvi AA. Incretin-based therapies, glucometabolic health and endovascular inflammation. Curr Pharm Des 2014; 20: 4953-60.

54. Rizzo M, Nikolic D, Patti AM, et al. GLP-1 receptor agonists and reduction of cardiometabolic risk: potential underlying mechanisms. Biochim Biophys Acta Mol Basis Dis 2018; 1864: 2814-21.

55. Amin EF, Rifaai RA, Abdel-Latif RG. Empagliflozin attenuates transient cerebral ischemia/reperfusion injury in hyperglycemic rats via repressing oxidative-inflammatoryapoptotic pathway. Fundam Clin Pharmacol 2020. doi: $10.1111 /$ fcp. 12548 .

56. Saint Luke's Health System. Dapagliflozin in respiratory failure in patients with COVID-19 (DARE-19). ClinicalTrials.gov website. https://www.clinicaltrials.gov/ct2/ show/NCT04350593. Accessed April 27, 2020.

57. Smith GD, Amos TA, Mahler R, Peters TJ. Effect of chloroquine on insulin and glucose homoeostasis in normal subjects and patients with non-insulin-dependent diabetes mellitus. Br Med J 1987; 294: 465-7.

58. Quatraro A, Consoli G, Magno M, et al. Hydroxychloroquine in decompensated, treatment-refractory noninsulin-dependent diabetes mellitus. A new job for an old drug? Ann Intern Med 1990; 112: 678-81.

59. American Diabetes Association. COVID-19: if you do get sick, know what to do. Accessed at https://www.diabetes.org/blog/coronavirus-covid-19-know-what-to-do on 30 March 2020.

60. Zhou J, Tan J. Diabetes patients with COVID-19 need better care. Metabolism 2020; 107: 154216. 